\title{
Acute physiological comparison of sub-maximal exercise on a novel adapted rowing machine and arm crank ergometry in people with a spinal cord injury
}

\author{
Bonita Sawatzky ${ }^{1,2}{ }^{凶}$, Brandon Herrington (D) $^{3}$, Kevin Choi (D) $^{3}$, W. Ben Mortenson (iD ${ }^{2,4}$, Jaimie Borisoff ${ }^{2,5}$, Carolyn Sparrey ${ }^{2,6}$ and \\ James J. Laskin ${ }^{2,7}$
}

(c) The Author(s), under exclusive licence to International Spinal Cord Society 2022

STUDY DESIGN: Non-randomized crossover trial.

OBJECTIVES: The objective of this study was to assess the oxygen uptake during exercise using the Adapted ROWing machine (AROW) compared to the more commonly used Arm Crank Ergometry (ACE) for people with spinal cord injury/disease (SCI/D) with or without trunk stability.

SETTING: Canada, Vancouver.

METHODS: Participants were from a convenience sample of 14 adults with SCI/D (age 21-63 y) which include those with lumbar to low cervical impairments currently exercising at least once per week using cardiovascular exercise equipment at our Physical Activity Research Centre. The interventions were non-randomized steady-state exercise bouts at self-selected low and moderate workloads on the AROW and ACE for 5 min each. Our primary outcomes were the rate of oxygen consumption ( $\mathrm{mL} / \mathrm{kg} / \mathrm{min}$ ) and the Borg 0-10 Rating Scale of Perceived Exertion (RPE).

RESULTS: A repeated measures two-way ANOVA $(p<0.05)$ indicated that exercising on the AROW resulted significantly greater oxygen consumption and perceived exertion than ACE at similar sub-maximal workloads which may be explained by the differences in efficiency between the devices (Partial eta squared $=0.84, F$ stat $=48.25$; Partial eta squared $=0.86, F$ stat $=53.54$ ). CONCLUSIONS: We have demonstrated that this form of upper extremity exercise had a greater RPE and $\mathrm{VO}_{2}$ on the $\mathrm{ACE}$ at a given workload. Thus, the AROW could provide a functional upper extremity workout that can be used for daily exercise for those with varying levels of $\mathrm{SCl}$.

Spinal Cord (2022) 60:694-700; https://doi.org/10.1038/s41393-022-00757-2

\section{INTRODUCTION}

Physical exercise plays a critical role in the management of chronic diseases and spinal cord injury/disease (SCI/D) SCI [1-3]. Exercise guidelines for adults with $\mathrm{SCl}$ indicate that cardiometabolic health benefits can be achieved with as little as $30 \mathrm{~min}$ of moderate to vigorous intensity aerobic exercise three times per week [4]. However, there are many barriers to exercise participation among people with $\mathrm{SCl}$, with a lack of time and lack of transportation to accessible facilities being the greatest barriers [5]. As a result, individuals with $\mathrm{SCl}$ are less active than the general population [6]. This inactivity has been exacerbated during the COVID-19 pandemic [7].

A lack of accessible facilities and equipment barriers to exercise have been reported among people with $\mathrm{SCl}$ [8]. Functional electrical stimulation (FES) is cited $[4,9,10]$ as an effective exercise modality for those with $\mathrm{SCl}$ but is expensive and often difficult to access in the community. Arm-crank ergometry (ACE) is a well- validated arm-only activity that increases exercise capacity in people with $\mathrm{SCl}$ [11]. This more accessible form of exercise primarily engages muscles of the anterior upper limbs [12, 13]. However, regular exercise with an ACE can exacerbate shoulder pain issues with increased internal shoulder rotation, head forward posture and kyphosis that are often seen populations that use manual wheelchairs for daily mobility $[14,15]$. Thus, an alternative and safer exercise option to obtain an effective exercise workload could be optimal.

Upper-body poling (Concept2 Ski-erg) (UBP) is a sport-specific training device that allows upper extremity exercise, and is popular in gym and clinical settings. A recent study using a graded exercise test protocol found that while the peak oxygen consumption $\left(\mathrm{VO}_{2 \text { peak }}\right)$ was not significantly different between UBP and ACE, the metabolic rate for the UBP at any given submaximal power output was $24 \%$ higher, across participants with or without a SCl. The cardiovascular response was similar however,

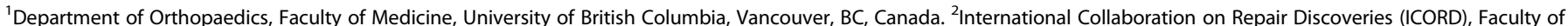



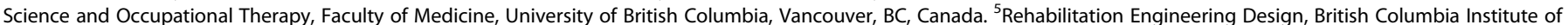


Rehabilitation Science, College of Health, University of Montana, Missoula, MT, USA. ${ }^{凶}$ email: bonita.sawatzky@ubc.ca

Received: 16 February 2021 Revised: 19 January 2022 Accepted: 20 January 2022

Published online: 3 February 2022 


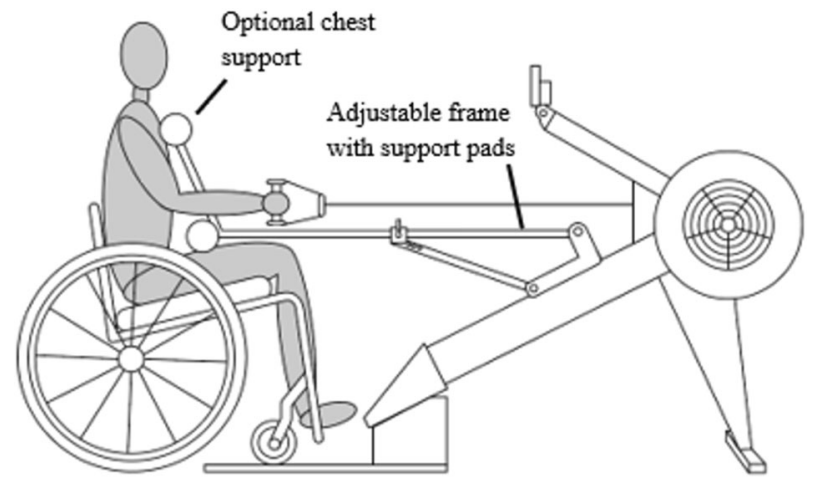

Fig. 1 Adapted Concept2 Rowing Ergometer (AROW) with optional trunk pad for trunk support.

the UBP peak power output was $19 \%$ lower than ACE possibly due to UBP's reduced efficiency. While the trunk was restrained, it is still possible that the UBP elicited a greater shoulder stabilizing response from the posterior shoulder and upper back musculature [16]. This can only be confirmed by EMG.

Another exercise option that has been adapted for people with $\mathrm{SCl}$ incorporates FES $[17,18]$ and rowing ergometry. FES rowing combines upper-body exercise with electrically stimulated lower body exercise, allowing an individual to row in a similar fashion to an able-bodied person. A recent systematic review showed FES rowing improved cardiovascular fitness and reduced bone density loss in persons with $\mathrm{SCl}[19,20]$. Activation of typically weaker and less used posterior shoulder muscles was observed with rowing [20]. Unfortunately, FES rowing ergometers, are expensive and less accessible [17].

The main challenge is the lack of accessible exercise equipment that people could have in their homes or local community centers. Another important factor to consider is not to exacerbate shoulder pain, common in this population with an exercise regime [14, 18]. Wheelchair users relies heavily on the anterior shoulder muscles thus an activity that focused on the posterior muscles would be favorable [21]. Rowing ergometers are less expensive now and have become popular in homes and community centers. For someone with a $\mathrm{SCl}$, a good quality $\mathrm{ACE}$ costs about three times the price of a rowing machine.

To address the issues of exercise equipment accessibility, functional and safe movement patterns and an exercise known to strengthen posterior shoulder and trunk/back muscles [18, 22], researchers at the International Collaboration for Repair Discovery's $\mathrm{SCl}$ research centre in Vancouver, British Columbia, Canada developed an Adapted ROWing Machine (AROW) [23]. The AROW was designed for people with or without trunk control who use manual or power wheelchairs. The AROW is set up using a Concept2 Model E Indoor Rower (Concept2, Inc, Morrisville, VT, USA) rowing ergometer with the monorail and glide seat removed. An adjustable frame with support pads was then mounted on the frame, and stability pads rest on the proximal thigh and upper chest of the user (Fig. 1). The chest support was optional and can be removed to maximize trunk flexion and extension for those with adequate trunk stability.

There are creative ideas for adapting a rower, such as (1) replacing the movable seat with a high-backed lockable seat, (2) removing the monorail and place the device on the floor, or (3) the ADAPT2Row which replaces the monorail with a small stand and knee pad to one would wheel up to (https://www.concept2. com/indoor-rowers/adaptive-rowing) [24]. However, none of these alternatives provide support for those individuals with impaired trunk control. AROW plans are open-source and available at https://adaptederg.commons.bcit.ca/.
In a recently published, qualitative study we explored the usability of the AROW as compared to the ACE [25]. Participants perceived the AROW to be the more enjoyable exercise and they felt it to be "a better workout" compared to the ACE. The objective of this paper was to compare the relative oxygen consumption $\left(\mathrm{VO}_{2}\right)$, and Rating of Perceived Exertion (RPE) at self-selected workloads while using the AROW compared to the ACE for people with $\mathrm{SCl} / \mathrm{D}$ including paraplegia and tetraplegia.

\section{HYPOTHESIS}

We hypothesized that at the same low and moderate individualized self-selected workloads the AROW would result in a similar or greater $\mathrm{VO}_{2}$ and RPE measurements as compared to the ACE.

\section{METHOD \\ Design}

This study used a non-randomized approach to compare the two ergometry modalities. This study included three components: orientation to the testing equipment, testing on the ACE (Lode Angio Rehab, Lode BV, Netherlands), and testing on the AROW. We used a similar study design used often in steady-state wheelchair propulsion studies, where the constant is velocity. Power output is then calculated to determine wheeling efficiency. In a recent paper on standard wheeling versus a backward row-like wheeling, velocity was constant and power output was measured [26]. Since velocity is meaningless to compare between ACE and AROW, we used workload as the constant instead. Two self-selected workloads were chosen by the participant: one low and one moderate. The workload selection was determined on the AROW and then that same output was dialed-in for the ACE. These workloads were then compared between the two exercise modalities. Figure 2 outlines the study flow for the two sessions, scheduled at the same time of day, (range 3-7 days apart). The order of the testing was non-randomized, alternating ACE or AROW first for sequential participants.

\section{Participants}

Inclusion criteria were those with $\mathrm{SCl} / \mathrm{D}$, full-time wheelchair (primary mode of indoor/outdoor mobility) users, over 18 years of age, wrote and spoke English, and performed cardiovascular exercise at least once a week at PARC (Table 1). PARC is a community-based research facility that examines the physical activity and health outcomes in people with SCI/D. All participants were screened using the Physical Activity Readiness Questionnaire [27]. Exclusion criteria were contraindications for exercise such as cardiovascular and respiratory illness. Participants were asked to abstain from caffeine prior to testing on the days of the study and asked to eat only a small meal $2 \mathrm{~h}$ prior to the testing sessions.

\section{Procedures}

Participant written informed consent was obtained prior to study participation. All aspects of the setup and testing were completed by a physical therapist/exercise physiologist. Ergometers were adjusted for comfort and to maximize the use of the upper extremities and the trunk. Assisted grips (Active Hands Company Limited, England) were provided, if necessary, for those with reduced grip strength. Pre-exercise blood pressure was taken at each session to ensure participant safety. Postexercise blood pressure was measured if the participant showed any signs of post-exercise stress or autonomic dysreflexia.

During the initial testing session, participant characteristic data were collected, participants were introduced to the equipment, testing protocols explained, and instruction in the use of the Borg 0-10 RPE scale [28] was completed. Participants were set up and practiced on the AROW first, identifying the two self-selected workloads using the 0-10 RPE scale as a guide that would then be used for testing on both the AROW and the ACE. The first workload determined was their warmup or light workload (2-3 RPE), and the second was their moderate effort (4-6 RPE) but one that could be sustained for at least $10 \mathrm{~min}$. The participant was coached that the workload on the AROW was determined by both the cadence of the rowing as well as how hard they pulled. While determining their selfselected workloads the researcher provided guidance and feedback to the participant to help ensure that the selected AROW workloads were submaximal. The workload and rowing cadence on the AROW were displayed 


\section{Session 1}

- Consent and equipment orientation

- Practice and self-selection of low (RPE 2-3) and moderate (RPE 4-6) workloads on the AROW

\section{Alternate* AROW OR ACE}

- Fitted with metabolic and HR monitor

- Warm up on AROW/ACE and practice at their selfselected low and moderate workloads

- Rest 5 minutes

- 5-minute test at self-selected low workload

- Rest 5 minutes

-5-minute test at self-selected moderate workload

\section{Session 2}

\section{Alternate* AROW OR \\ ACE}

- Fitted with metabolic and HR monitor

- Warm up on AROW/ACE and practice at their selfselected low and moderate workloads

- Rest 5 minutes

-5-minute test at self-selected low workload

- Rest 5 minutes

- 5-minute test at self-selected moderate workload

Fig. 2 Testing session flow chart. *The order of the actual testing was alternated with the first participant being tested on the AROW during session 1 and the ACE during session 2 with the following participant being tested first on the ACE and then on the AROW during the second session.

Table 1. Individual characteristics of participants.

\begin{tabular}{|c|c|c|c|c|c|}
\hline Participant & Functional/Injury level & ASIA Impairment Scale & Injury duration (years) & Weight (kg) & Height $(\mathrm{cm})$ \\
\hline 1 & High $\mathrm{T}$ & A & 30 & 62.3 & 180 \\
\hline 2 & $\mathrm{~L}$ & $B$ & 16 & 72.1 & 177.8 \\
\hline 3 & Mid T & A & 3 & 57.6 & 170.2 \\
\hline 4 & C & B & 23 & 65.3 & 170.2 \\
\hline 5 & High T & A & 35 & 77.1 & 162.6 \\
\hline 6 & High T & A & 17 & 68 & 177.8 \\
\hline 7 & High $\mathrm{T}$ & A & 2 & 70.3 & 175.3 \\
\hline 8 & Low $T$ & A & 9 & 133.8 & 193 \\
\hline $9^{a}$ & C & NA & $30+$ & 82.6 & 182.9 \\
\hline $10^{a}$ & $L$ & B & 35 & 81 & 154.9 \\
\hline 11 & Low $\mathrm{T}$ & A & 3 & 95 & 188 \\
\hline 12 & High T & A & 3 & 76.4 & 177.8 \\
\hline 13 & C & A & 24 & 47.6 & 180.3 \\
\hline 14 & C & A & 10 & 79.4 & 190.5 \\
\hline
\end{tabular}

Classification is based on the criteria established by the American Spinal Injury Association.

A complete injurym $B$ sensory incompletem $C$ cervicalm $T$ thoracicm $L, N A$ not applicable (exact lesion levels and sex were not provided to maintain anonymity).

${ }^{a}$ Non-traumatic. 
on the device's performance monitor and were recorded every $15 \mathrm{~s}$. The participant was instructed to watch the AROW's performance monitor and do their best to maintain their cadence and workload. When using the ACE ergometer, it was set to its constant power mode. In this mode the researcher pre-set the device to the desired workload (matching the predetermined AROW workloads), thus regardless of the participant's cranking cadence the workload remained the same. Therefore, it was unnecessary for the participant or researcher to monitor cadence or effort while using the ACE ergometer. The physiological testing of the AROW and ACE ergometers followed identical protocols (see Fig. 2) using a Parvo Medics TrueOne 2400 (Salt Lake City, USA) metabolic with facemask and a Polar HR monitor, (Polar Electro Oy; Kempele, Finland).

\section{Outcome measures}

Primary outcome: Sub-maximal low and moderate $\mathrm{VO}_{2}$

Steady-state, breath-by-breath metabolic data was recorded every $15 \mathrm{~s}$ during each of the four 5-minute exercise bouts.

Secondary outcome: RPE

The Borg Scale for RPE was used to record the perceived physical exertion after exercising on the ergometers. Prior to testing, we explained the scale and the verbal expressions used to anchor perception of exertion to specific ratings.

Weight was recorded to the nearest $0.1 \mathrm{~kg}$ using a wheelchair scale. Height was recorded to the nearest $0.1 \mathrm{~cm}$ with the participant supine. The level, classification, and injury duration of $\mathrm{SCl}$ were self-reported by participants.

\section{Data analysis}

The relative $\mathrm{VO}_{2}(\mathrm{ml} / \mathrm{kg} / \mathrm{min})$, heart rate $(\mathrm{bpm})$ and workload $(\mathrm{W})$ were averaged over the last 60 seconds of the given testing session for analysis. The RPE was assessed at the completion of each testing session. Efficiency was calculated, described by Baumgart, et al., [16] as the ratio of workload to metabolic rate (WL/MR). A repeated measures two-way ANOVA $(p<$ 0.05 ) was used to assess the effect of the exercise modalities and exercise intensities on our primary outcome, relative $\mathrm{VO}_{2}$ and secondary outcome, RPE. A two-tailed paired Student $t$-test $(p<0.05)$ was used to compare the effect of exercise modality with low-intensity exercise only on $\mathrm{VO}_{2}$ and RPE. Normality of distributions were verified using the Shapiro-Wilk and the Kolmogorov-Smirnov tests. All statistical analyses were completed using IBM SPSS Statistics 26. GraphPad Prism 8.4.2 was used to create beforeafter plots.

\section{RESULTS}

The means and standard deviations self-selected low intensity and moderate intensity workload were significantly different $(F(1,9)=$ $26.2 ; p<0.005$ ), $24.4 \pm 18.8 \mathrm{~W}$ (range $=5-70 \mathrm{~W}$ ) and $39 \pm 20.7$ (range $=15-75 \mathrm{~W})$, respectively. All participants were able to complete the low intensity testing bouts. Due to the relative difficulty, four participants could not complete the tests on the AROW at a moderate intensity and stay within the RPE guidelines of 4-6. Therefore, only 10 participants were included in the twoway ANOVA (Table 2). There were no reported complaints or adverse effects from the participants during the study.

\section{Comparison of ergometry method for relative oxygen consumption $\left(\mathrm{VO}_{2}\right)$}

Based on the tests for normality, there were no departures of normality for our primary outcome variable, $\mathrm{VO}_{2}$, for either the twoway RM ANOVA or paired $t$-tests. However, for the secondary subjective measure of RPE, this assumption was breached with both normality tests for low intensity but not high. The mean $\mathrm{VO}_{2}$ for AROW at low and moderate intensities was $12.0 \pm 4.1 \mathrm{~mL} / \mathrm{min} / \mathrm{kg}$ and $14.9 \pm 4.6 \mathrm{~mL} / \mathrm{min} / \mathrm{kg}$, respectively. Mean $\mathrm{VO}_{2}$ for ACE at low and moderate intensities was $9.0 \pm 3.7 \mathrm{~mL} / \mathrm{min} / \mathrm{kg}$ and $11.2 \pm 4.7$ $\mathrm{mL} / \mathrm{min} / \mathrm{kg}$, respectively. Using the repeated measures two-way ANOVA, we found no significant interaction between the effects of exercise modality and exercise intensity on $\mathrm{VO}_{2}(\mathrm{~F}(1,9)=3.222$; $p=0.106$ ). Main effect of exercise modality on $\mathrm{VO}_{2}$ indicated a significantly higher oxygen uptake using the AROW compared to the ACE (Partial eta squared $=0.84 ; F=48.25 ; p<0.001$ ). Main effect of exercise intensity on $\mathrm{VO}_{2}$ indicated a significant difference between the low and moderate intensities exercise (Partial eta squared $=0.76 ; \mathrm{F}=27.9 ; p<0.001)$. We conducted paired t-tests assessing the effect of exercise modality at low intensity exercise only on $\mathrm{VO}_{2}$ and RPE to include the four participants that were excluded from the two-way ANOVA (Fig. 3). We found a significantly greater $\mathrm{VO}_{2}$ using the AROW $(11.4 \pm 3.7 \mathrm{~mL} / \mathrm{min} / \mathrm{kg})$ compared to the ACE $(8.6 \pm 3.2 \mathrm{~mL} / \mathrm{min} / \mathrm{kg})$ ergometry $(t=6.2, p<0.001)$. Gross efficiency for the ACE vs the AROW were $10.1 \%$ and $7.3 \%$, respectively for low intensity and $13.5 \%$ and $9.8 \%$ for moderate intensity. Individual physiological measurements and means are summarized in Table 2.

\section{Comparison of ergometry method for perceived exertion (RPE)}

Mean RPE for AROW at low and moderate steady-state workloads was $3.5 \pm 1.7$ and $5.8 \pm 1.9$, respectively. Mean RPE for ACE at low and moderate workloads was $1.9 \pm 1.2$ and $3.5 \pm 1.4$, respectively. Using the repeated measures two-way ANOVA, we found a significant interaction between the effects of exercise modality and exercise workloads for $\operatorname{RPE}(F=6.311 ; p=0.033)$. Main effect of exercise modality on RPE indicated a higher RPE for the AROW over the ACE (Partial eta squared $=0.86 ; F$ stat $=$ 53.54; $p<0.001$ ). Main effect of workloads on RPE indicated a significant difference between low and moderate intensity exercise (Partial eta squared $=0.85 ; F$ stat $=49.7 ; p<0.001$ ). We also found a significantly higher RPE for the AROW $(3.9 \pm 1.8)$ compared to the ACE $(2.5 \pm 1.8)$ ergometer $(t(13)=3.0 ; 95 \% \mathrm{Cl}$ $[0.41,2.52] ; p<0.010)$ at low intensity which was even greater at the moderate intensity level $(p<0.001)$, see Fig. 3 .

\section{DISCUSSION}

This novel study describes the physiological benefits of a non-FES rowing exercise for participants with $\mathrm{SCl}$ with paraplegia and tetraplegia. It showed that for a given workload, participants required a significantly greater $\mathrm{VO}_{2}$ on the AROW than they did on the ACE with large effect sizes $(>0.80)$. These results were possibly due to two factors. One factor was the decreased efficiency of the rowing machine, requiring more effort for a given workload. ACE biomechanics allows for a limited degree of freedom of movement; therefore, power can be put directly into the crank system, resulting in $3 \%$ greater efficiency. The other factor may be that rowing uses more of the larger posterior shoulder muscles, possibly increasing the energy demand of co-contractions. In our early unpublished data comparing muscle activation time between AROW and ACE, on average muscles were "on" AROW $9 \%$ longer for nine upper extremity muscles for one individual [29]. This latter theory needs more evidence, however, to support it.

This difference in efficiency was also found in the study by Baumgart et al. [16] when comparing the UBP and the ACE. While the $\mathrm{VO}_{2}$ peak found between the devices was not significantly different, the $\mathrm{VO}_{2}$ at any given sub-maximal workload was significantly higher for the UBP. Like in the current study this discrepancy is a result of a lower efficiency as both the UBP and the AROW provide a much less constrained pattern of movement as compared to the ACE [16]. Fortunately, the purpose of an exercise ergometer is not necessarily to produce power efficiently, but rather to provide its user with a cardiovascular workout.

Secondly, this study found that exercising on the AROW was perceived as being more difficult than exercising on the ACE based on the RPE. This observation is consistent with physiological data obtained during the study. Since $\mathrm{VO}_{2}$ measured during AROW was higher than during ACE, logic follows that RPE would also be higher as their oxygen consumption was increased. Additionally, the ACE was set up at a predetermined workload. That is, participants could cycle at a self-selected pace and the 


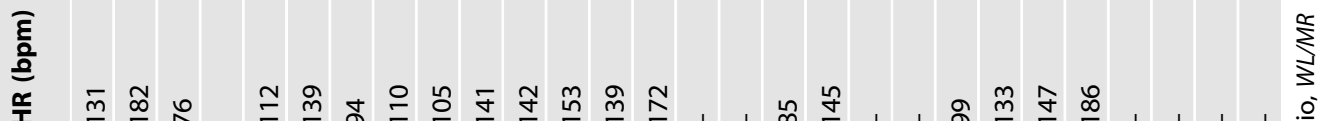

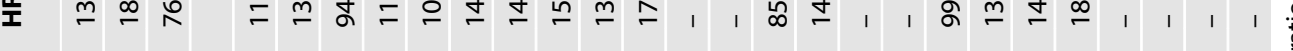

文

३)

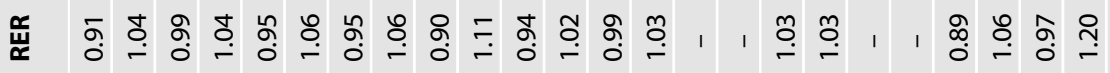

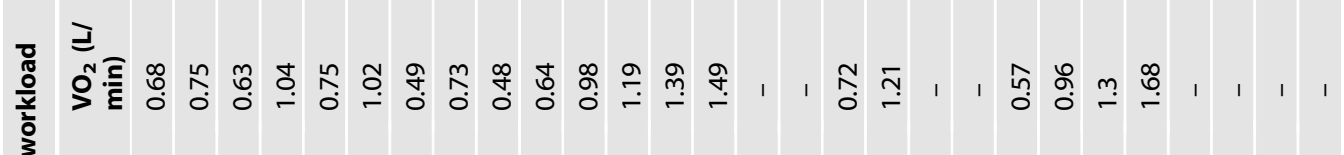

崖

$\frac{u}{\frac{u}{x}}$



这

$\sum$

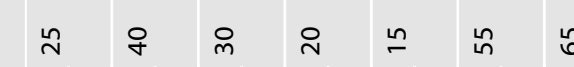



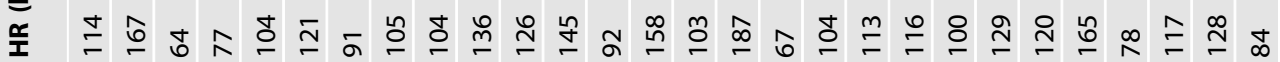



$\stackrel{2}{\circ}$

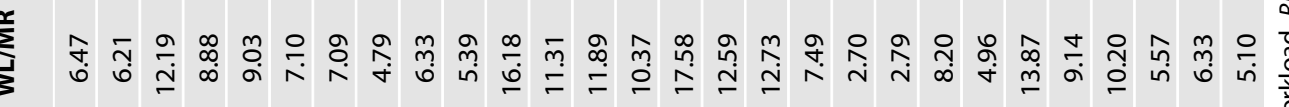

« 영

.

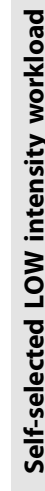

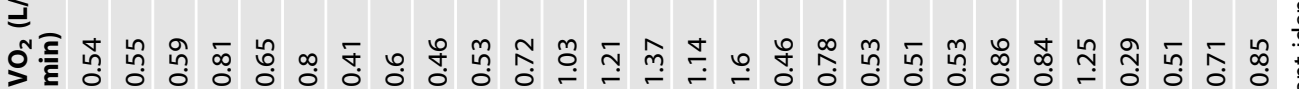

हे



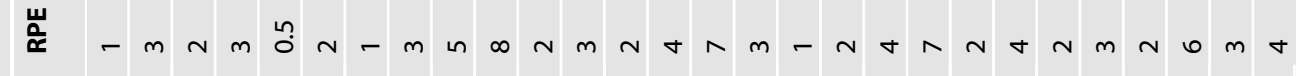

$\sum$

$\simeq$ ก

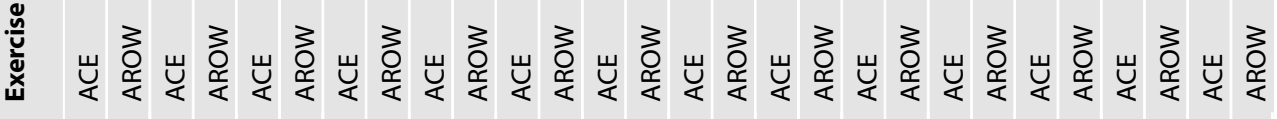

$\underline{0}-$

m

$\checkmark$

in

$\circ$

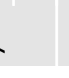

$\infty$

$\therefore=\stackrel{m}{ }$ 

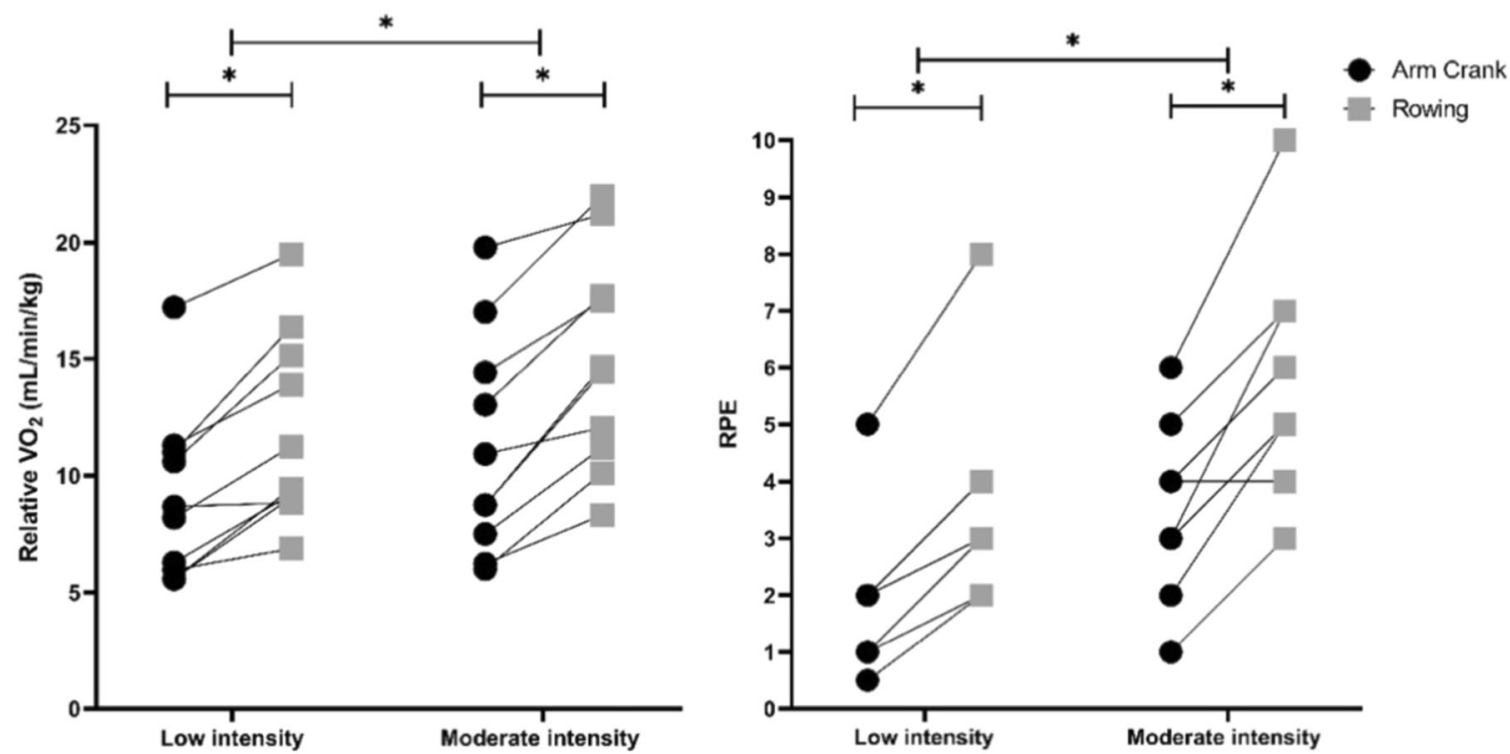

Fig. 3 Effect of exercise modality and exercise intensity on relative $\mathbf{V O}_{2}$ and RPE. Dark circles represent arm crank ergometer. Gray boxes represent adapted rower. ${ }^{*} p<0.001$ between modalities and intensities.

machine resistance would compensate to ensure the appropriate workload was obtained. During AROW use, participants had to follow a specific pulling cadence and strength to obtain the desired workload. This latter issue may have been the reason they perceived the workout to be more difficult on the moderate intensity as seen by an interaction effect. The cognitive demand is likely to be higher on the AROW.

With a higher RPE being observed on the AROW compared to the $A C E$, as well as four participants not being able to complete the moderate rowing difficulty level, one question that arose was whether people with SCI/D would choose to exercise on the AROW in the community if given the chance. This question was addressed by the companion qualitative research examining the preferences of the current study cohort [25]. Thirteen participants indicated that the AROW could be better for cardiovascular exercise compared to the ACE, citing working more muscle groups, challenging, ability to raise heart rate, and backward pulling motion as contributing factors to the better workout. Twelve participants reported they would use the AROW in the community. This qualitative study showed that participants did not perceive the higher RPE on the AROW negatively [25].

The study included a heterogeneous population with a variety of spinal cord lesion levels, age, weight, and activity levels represented increasing the generalizability, however, the study cohort was likely more active than the population of people with $\mathrm{SCl} / \mathrm{D}$ at large since our criteria was participants who exercised at least once per week. The relatively large range of injury levels and performance (workloads) that could be achieved, suggests that it is not just for those with paraplegia but those with limited trunk control can use the AROW. Our small sample does increase the risk for a Type II error. However, we purposefully focused on whether the results could achieve a large effect size on the primary outcome variable, which it did. The values obtained here will be useful to calculate sample size for a future larger intervention.

Previous studies on FES rowing and fixed-seat rowing have used electromyography data to show activation of posterior shoulder and scapular stabilizer muscles. The benefit of performing a rowing exercise for fitness is that muscles already used for wheeling are now being balanced by strengthening the agonist posterior muscles. Rowing ergometry allows repeated activation of the posterior shoulder and scapular stabilizers - both of which are not typically used during wheelchair propulsion [30].
Improvement of shoulder strength and scapular stability is important in preventing and treating shoulder problems, and strengthened truncal musculature aids in balance and functionality, including benefits in seated stability, posture, and reach [29]. Assumptions can be made that the AROW also activates these muscles, but no electromyographic data was obtained during the study to demonstrate this definitively. Further research to study muscle activation patterns during AROW compared to ACE, and to explore the physiological differences between the two during an extended fitness regime will be the next steps in the research program.

Finally, exercise in the present study was confined to only $5 \mathrm{~min}$ of AROW use. No comparisons were made between ACE and AROW over a longer duration of use. Further research should explore how long participants can use the AROW and whether they will experience any unexpected overuse injuries from this activity. Possible concerns included skin shearing from micromotions at the seat or support pads with longer duration and frequency of AROW use. To address the above limitations, our future studies with larger samples will: (1) explore those individuals with $\mathrm{SCI} / \mathrm{D}$ not currently engaged in regular exercise, (2) examine AROW use over an extended period and duration, and (3) quantify physiological and psychological costs and benefits of using such a device within a community gym compared to a home program since lower cost systems are more readily available to adapt. The recent isolation issues during the pandemic highlighted the importance of those who use wheelchairs and their access to cardiovascular exercise, thus needing affordable home options to keep fit. We are currently working on an affordable home model.

This is the first study looking at an adaptive-rowing exercise without FES. Otherwise, there is only a protocol paper published proposing a randomized control trial using the ADAPT2row device [24]. In addition, the novelty of our study was testing of an innovative adaptation to a standard rowing machine that would facilitate its use by those living with an SCl. Modifying the rower provided another way of exercising that did not require a specialized centre that houses arm crank ergometers. We have demonstrated that this form of upper extremity exercise resulted in greater RPE and $\mathrm{VO}_{2}$ than the ACE at a given workload. The AROW could provide a functional upper extremity workout that can be used for daily exercise for those with varying levels of $\mathrm{SCl}$. 


\section{DATA AVAILABILITY}

All data generated or analyzed during this study are included in this published article.

\section{REFERENCES}

1. Cragg JJ, Noonan VK, Krassioukov A, Borisoff J. Cardiovascular disease and spinal cord injury: results from a national population health survey. Neurology. 2013;81:723-8.

2. Pattyn N, Pattyn N, Cornelissen VA, Cornelissen VA, Eshghi SRT, Eshraghi SRT, et al. The effect of exercise on the cardiovascular risk factors constituting the metabolic syndrome: a meta-analysis of controlled trials. Sports Med. 2013;43:121-33.

3. Jacobs PL, Nash MS. Exercise recommendations for individuals with spinal cord injury. Sports Med. 2004;34:727-51.

4. Martin Ginis KA, Van Der Scheer JW, Latimer-Cheung AE, Barrow A, Bourne $C_{\text {, }}$ Carruthers $P$, et al. Evidence-based scientific exercise guidelines for adults with spinal cord injury: an update and a new guideline. Spinal Cord. 2018;56:308-21.

5. de Groot S, Kouwijzer I, Valent L, Hagoort M, Ten Hoorn A, van der Woude L, et al. Sport participation after the HandbikeBattle: benefits, barriers, facilitators from the event-a follow-up survey. Spinal Cord Ser Cases. 2020;6:54-54.

6. Myers J, Lee M, Kiratli J. Cardiovascular disease in spinal cord injury: an overview of prevalence, risk, evaluation, and management. Am J Phys Med Rehabil. 2007;86:142-52.

7. Marco-Ahulló A, Montesinos-Magraner L, González L-M, Morales J, BernabéuGarcía JA, García-Massó X. Impact of COVID-19 on the self-reported physical activity of people with complete thoracic spinal cord injury full-time manual wheelchair users. J Spinal Cord Med. 2021:1-5. https://doi.org/10.1080/ 10790268.2020.1857490.

8. van den Akker LE, Holla JFM, Dadema T, Visser B, Valent L, de Groot S, et al. Determinants of physical activity in wheelchair users with spinal cord injury or lower limb amputation: perspectives of rehabilitation professionals and wheelchair users. Disabil Rehabil. 2019;42:1-8.

9. Hettinga DM, Andrews BJ. Oxygen consumption during functional electrical stimulation-assisted exercise in persons with spinal cord injury. Sports Med. 2008;38:825-38.

10. Sadowsky CL, Hammond ER, Strohl AB, Commean PK, Eby SA, Damiano DL, et al. Lower extremity functional electrical stimulation cycling promotes physical and functional recovery in chronic spinal cord injury. J Spinal Cord Med. 2013;36:623-31.

11. Pelletier CA, Ditor DS, Latimer-Cheung AE, Warburton DE, Hicks AL. Exercise equipment preferences among adults with spinal cord injury. Spinal Cord. 2014;52:874-9.

12. Faupin A, Gorce $P$, Watelain E, Meyer C, Thevenon A. A biomechanical analysis of handcycling: a case study. J Appl Biomech. 2010;26:240-5.

13. Litzenberger S, Mally F, Sabo A. Biomechanics of elite recumbent handcycling: a case study. Sports Eng. 2016;19:201.

14. Ballinger DA, Rintala DH, Hart KA. The relation of shoulder pain and range-ofmotion problems to functional limitations, disability, and perceived health of men with spinal cord injury: a multifaceted longitudinal study. Arch Phys Med Rehabil. 2000;81:1575-81.

15. Quittmann OJ, Meskemper J, Abel T, Albracht K, Foitschik T, Rojas-Vega S, et al. Kinematics and kinetics of handcycling propulsion at increasing workloads in able-bodied subjects. Sports Eng. 2018;21:283-94.

16. Baumgart JK, Gurtler L, Ettema G, Sandbakk O. Comparison of peak oxygen uptake and exercise efficiency between upper-body poling and arm crank ergometry in trained paraplegic and able-bodied participants. Eur J Appl Physiol. 2018;118:1857-67.

17. Wheeler GD, Andrews B, Lederer R, Davoodi R, Natho K, Weiss $C$, et al. Functional electric stimulation-assisted rowing: Increasing cardiovascular fitness through functional electric stimulation rowing training in persons with spinal cord injury. Arch Phys Med Rehabil. 2002;83:1093-9.

18. Wilbanks SR, Rogers R, Pool S, Bickel CS. Effects of functional electrical stimulation assisted rowing on aerobic fitness and shoulder pain in manual wheelchair users with spinal cord injury. J Spinal Cord Med. 2016;39:645-54.

19. Ye G, Grabke EP, Pakosh M, Furlan JC, Masani K. Clinical benefits and system design of FES-rowing exercise for rehabilitation of individuals with spinal cord injury: a systematic review. Arch Phys Med Rehabil. 2021;102:1595-905.

20. Olenik LM, Laskin JJ, Burnham R, Wheeler GD, Steadward RD. Efficacy of rowing, backward wheeling and isolated scapular retractor exercise as remedial strength activities for wheelchair users: application of electromyography. Paraplegia. 1995;33:148.
21. Liampas A, Neophytou P, Sokratous M, Varrassi G, loannou C, Hadjigeorgiou G, et al. Musculoskeletal pain due to Wheelchair use: a systematic review and meta analysis. Pain Ther. 2021;10:973-84.

22. Shaharudin S, Zanotto D, Agrawal S. Muscle synergies of untrained subjects during 6 min maximal rowing on slides and fixed ergometer. J Sports Sci Med. 2014;13:793-800.

23. Mattie J. Adaptations to support independent use of commercially available rowing ergometers by people with spinal cord injuries. The 36th International Seating Symposium; 2020; Vancouver, British Columbia, Canada, 2020.

24. Hansen RK, Samani A, Laessow U, Handberg A, Larsen RG. Effect of wheelchairmodified rowing exercise on cardiometablic risk factors in spinal cord injured wheelchair users: Protocol for a randomized controlled trial. BMJ Open. 2020;10:3040727.

25. Wong R, Stewart A, Mortenson W, Sawatzky B, Laskin J, Borisoff J, et al. Exploring exercise participant and the usability of the Adaptive Rower and Arm Crank Ergometer through wheelchair users' perspectives. Disabil Rehabil. 2021. https:// doi.org/10.1080/09638288.2021.1894245

26. Habibi A, MacGillivray M, Kalra H, Sawatzky B. Efficiency and perceived exertion of manual wheelchair propulsion: a physiological comparison of push vs pull wheeling. J Med Eng Technol. 2021;45:249-57.

27. Warburton DER, Jamnik V, Bredin SSD, Shephard RJ, Gledhill N. The 2018 Physical Activity Readiness Questionnaire for Everyone (PAR-Q+) and electronic Physical Activity Readiness Medical Examination (ePARmed-X+). Health Fit J Can. 2018;11:31-34.

28. Borg G. Borg's Perceived exertion and pain scales. Champaign, IL: Human Kinetics; 1998.

29. Behrouzian M, Laskin J, Newell R, Mortenson B, Borisoff J, Sawatzky B. Muscle activation of adapted ergometry rowing and cycling for people with $\mathrm{SCl}$. Presented at Undergraduate Medical Education Orthopaedics Research Day. Vancouver, Canada: University of British Columbia; 2021.

30. Paralyzed Veterans of America Consortium for Spinal Cord M. Preservation of upper Li function following spinal cord injury: a clinical practice guideline for health-are professionals. J Spinal Cord Med. 2005;28:434-70.

\section{AUTHOR CONTRIBUTIONS}

$\mathrm{BS}$ and $\mathrm{JL}$ were responsible for conceiving this article, overseeing the data collection, analyses, supervising the writing, editing, and revisions of the manuscript. $\mathrm{BH}$ and $\mathrm{KC}$ conducted the analyses and were key contributors to the writing of the manuscript. BM provided input, reviewed, and edited manuscript. JB had a role in initiating the project with BS. He was key to developing the AROW and determining the study protocol. He reviewed and edited manuscript. CS had a significant role in initiating the project and key to developing the AROW. She reviewed and edited manuscript.

\section{FUNDING}

Financial assistance was received in support of this research study by the Rick Hansen Institute. The AROW was previously funded by the Craig Nielsen Foundation.

\section{COMPETING INTERESTS}

The authors declare no competing interests.

\section{ETHICAL APPROVAL}

We certify that all applicable institutional and governmental regulations concerning the ethical use of human volunteers/animals were followed during this research.

\section{ADDITIONAL INFORMATION}

Correspondence and requests for materials should be addressed to Bonita Sawatzky.

Reprints and permission information is available at http://www.nature.com/ reprints

Publisher's note Springer Nature remains neutral with regard to jurisdictional claims in published maps and institutional affiliations. 